\title{
The effect of ACE2 inhibitor MLN-4760 on the interaction of SARS-CoV-2 spike protein with human ACE2: a molecular dynamics study
}

\author{
Babak Nami ${ }^{1,2, *}$, Avrin Ghanaeian ${ }^{1,2}$, Kasra Ghanaeian ${ }^{2,3}$, Negin Nami $^{2}$ \\ ${ }^{1}$ Department of Medical Genetics, University of Alberta, Edmonton, AB, Canada \\ ${ }^{2}$ COVID-19 Research Group \\ ${ }^{3}$ School of Medicine, Gonabad University of Medical Sciences, Gonabad, Iran \\ *Correspondance: namimoll@ualberta.ca; (Twitter: BabakNami)
}

\section{ABSTRACT}

A previous study shows that an ACE2 enzymatic activity inhibitor efficiently blocks the interaction of SARS-CoV spike protein with human ACE2 and may be effective in preventing the coronavirus membrane fusion and entry to human cells. The report suggests that potent ACE2 inhibitors can be used to treat hypertension as well as for controlling SARS-CoV infection. We here studied the effect of a selective and highly potent ACE2 inhibitor (MLN-4760) on the interaction of the SARS-CoV-2 spike receptor-binding domain (RBD) with human ACE2 by molecular dynamics (MD) simulation. To this end, we docked the RBD of SARS-CoV-2 to the human native ACE2 and the ACE2 complexed with MLN-4760, and analyzed the dynamics, protein-protein and ligand-protein interactions of the complexes by MD simulation in a simulated biological condition for 100 ns. Analyzing crystallographic structures of SARS-CoV-2 and SARSCoV RBDs in the complexes with human ACE2 showed that RBD of SARS-CoV-2 binds to ACE2 with a higher affinity than that of SARS-CoV. Results also revealed that MLN-4760 binds to ACE2 at the enzymatic active site with a high affinity and significantly alters the ACE2 protein conformation. MLN-4760 also changes the binding site and the residues involved in hydrogen and hydrophobic binding between RBD and ACE2, however, it had no major effect on the binding affinity of the interaction between RDB and ACE2. Interestingly, binding RBD to the ACE2 complexed with MLN-4760 abrogated the inhibitory effect of MLN-4760 and rescued the conformation of the ACE2 enzymatic site by reforming the closed conformation to the open native conformation. This was due to the disassociation of MLN-4760 from the enzymatic active site of the ACE2 in the result of RBD binding. Overall, these results show that MLN-4760 does neither block nor increase the binding of SARS-CoV-2 spike RBD to human ACE2 and probably had no effect on the viral entry. However, binding the spike protein to ACE2 can rescue the enzymatic function of ACE2 from its inhibitor.

Keywords: COVID-19; SARS-CoV-2; ACE2; Spike protein; MLN-4760; Inhibitor; Molecular dynamics 


\section{INTRODUCTION}

Severe acute respiratory syndrome coronavirus 2 (SARS-CoV-2) earlier named as 2019$\mathrm{nCoV}$ is the virus strain that causes severe acute respiratory syndrome (SARS) called COVID19 disease that first identified in December 2019 in Wuhan city of Hubei province, China [1-4]. COVID-19 is a contagious acute respiratory infectious disease clinically characterized by symptoms as fever, headache, dry cough, shortness of breath, sore throat, fatigue, and less commonly abdominal pain, muscle pain, diarrhea, and loss of smell [5-8]. Some infections result in viral pneumonia and multi-organ failure and even progress to death [5-8]. Following the rapid global outbreak, the World Health Organization declared COVID-19 a pandemic on March 11, 2020 [9]. As of 20 April 2020, more than 2.4 million confirmed COVID-19 cases have been reported across 210 countries and territories, causing over 165,000 deaths [10].

SARS-CoV-2 belongs to the Coronaviridae family of viruses that cause common cold to severe respiratory diseases in humans. SARS-CoV-2 is a member of subgenus Sarbecovirus (beta-CoV lineage B) and is closely related to the coronaviruses caused SARS and Middle East Respiratory Syndrome (MERS) outbreak in the past two decades [11,12]. SARS-CoV-2 has a linear positive-sense single-stranded RNA (+ssRNA) genome with approximately 30,000 nucleotides in length [11,12]. The genome encodes for ten open reading frames (ORFs) including four viral structural proteins; spike protein (S), an envelope protein (E), membrane protein $(M)$, and nucleocapsid phosphoprotein $(N)$. The $S, M, E$ proteins form the viral coat, and the $\mathrm{N}$ protein is involved in the packaging of the viral RNA genome [13-15]. The Non-structural proteins include ORF1ab, ORF3a, ORF6, ORF7a, ORF10, and ORF8. ORF1ab encodes RNA-dependent RNA polymerase polyprotein and is responsible for transcription and replication of viral RNAs [13]. SARS-CoV-2 share $80-96 \%$ and $92-99 \%$ genetic material with the coronavirus strains derived from bats and pangolin indicating SARS-CoV-2 to have a zoonotic origin [16-21]. The non-structural proteins are highly homologous to the bat coronavirus and have significant homology with the SARS-CoV [6,11-15]. However, the spike protein, ORF8 and ORF3a proteins are considerably different from other known SARS-like coronaviruses, and they may cause more transmission rate than SARS-CoV [6,11-15].

SARS-CoV-2 enters human epithelial cells through the binding of the spike protein from its $\mathrm{N}$ terminal receptor-binding domain (RBD) to the angiotensin I converting enzyme 2 (ACE2) 
receptor on the host cell surface [13-15,22,23] (Fig 1A). ACE2 enzyme belongs to the reninangiotensin peptide hormone system is a necessary regulator for cardiovascular physiology and is highly expressed in the lungs, arteries, heart, kidney, and intestines and [24,25]. ACE2 catalyzes the cleavage of angiotensin I peptide into vasodilator angiotensin 1-9, and angiotensin II peptide into the vasodilator angiotensin 1-7 and play role in controlling blood pressure [25]. Renin-angiotensin system blockers including ACE inhibitors (ACEls) and angiotensin receptor blockers (ARBs) are highly used drugs for the treatment of patients with cardiovascular diseases including heart attack, hypertension, chronic kidney disease (nephropathy) and diabetes [26].

Blocking the binding of SARS-CoV-2 spike proteins to ACE2 receptors on the human cell is the first and most promising approach for blocking cell entry and inhibiting SARS-CoV-2 infection. In the year 2004, Huentelman et al. [27] introduced N-(2-aminoethyl)-1 aziridineethanamine (NAAE; $\mathrm{C}_{6} \mathrm{H}_{15} \mathrm{~N}_{3}$ ) as a novel human ACE2 inhibitor which blocks the attachment of SARS-CoV spike protein to ACE2 receptor. The report suggests that NAAE can prevent SARSCoV entry to ACE2 expressing human cells and the potential to be used as an anti-SARS agent [27]. In this study, we aimed to investigate whether an enzymatic inhibitor of ACE2 is able to affect the interaction of SARS-CoV-2 spike protein with ACE2. Here, we study the effect of a selective and highly potent ACE2 inhibitor (S,S)-2-\{1-Carboxy-2-[3-(3,5-dichloro-benzyl)-3Himidazol-4-YL]-ethylamino\}-4-methyl-pentanoic acid (MLN-4760; $\mathrm{C}_{19} \mathrm{H}_{23} \mathrm{C}_{12} \mathrm{~N}_{3} \mathrm{O}_{4}$ ) on the binding of SARS-CoV-2 spike RBD to ACE2.

\section{METHODS}

\section{Model structures}

Crystallographic structures of SARS-CoV-2 spike RBD (PDB 6LZG), native human ACE2 (PDB 1R42 [28]) and human ACE2 complexed with NLM-4760 at the enzymatic active site (PDB 1R4L [28]) were obtained from Protein Data Bank (PDB). To dock the spike RBD to the ACE2 structures, the ACE2 structures topologically aligned to the 6LZG model by MatchMaker command in UCSF Chimera software using the coordinate of ACE2 from 6LZG model as a reference. Duplicated atoms were deleted, the model structures were combined and then PDB files of the hybrid complexes were generated.

\section{Molecular dynamics (MD) simulation}


MD simulation was performed using GROMACS version 2018 [29] and CHARMM 36 all-atom force field [30]. The structures were solvated in a cubic water box with a dimension that the protein structures were placed in with one nanometer $(\mathrm{nm})$ distance from side filled by single point charge water molecules as solvent according to the TIP3P water model [31]. The system charge was neutralized by $\mathrm{Cl}^{-} \mathrm{Na}^{+}$ions. Energy minimization was done with the steepest descent algorithm in 5,000 steps to eliminate inappropriate contacts between amino acids. The non-hydrogen atoms of the ligands were restrained, and system equilibration was done in two steps including NVT (constant volume of particles, volume, and temperature) and NPT (constant pressure of particles, volume, and temperature) ensembles in $1 \mathrm{~ns}$ at $300 \mathrm{~K}\left(37^{\circ} \mathrm{C}\right)$ and 1 atmosphere pressure. MD simulation was done for $100 \mathrm{~ns}$ at $300 \mathrm{~K}$. All the covalent bonds were constrained by using the Linear Constraint Solver (LINCS) algorithm. The Particle-Mesh Ewald (PME) summation method with a grid spacing of $0.16 \mathrm{~nm}$ and Van der Waals interactions with $1.2 \mathrm{~nm}$ cutoff was used to describe long-range electrostatic interactions. The simulations were performed with a 2-femtosecond (fs) timestep for 100 ns using Leapfrog integrator in the Cedar supercomputer.

\section{RESULTS AND DISCUSSION}

\section{SARS-CoV-2 spike RBD binds to human ACE2 with higher affinity compared to SARS- CoV}

Alignment of spatial structure and amino acid sequence of spike RBD of SARS-CoV-2 (PDB 6LZG) to those of SARS-CoV (PDB: 3SCI) and MERS-CoV (PDB 6C6Z) revealed a significant topologic and sequence difference between the spike RBD of SARS-CoV-2 and those of other two CoVs (Figs 1B and C). The similarity between the spike RBD of SARS-CoV-2 and that of SARS-CoV was found $74.4 \%$ (166 amino acids), while only $17.9 \%$ (40 amino acid) similarity was found between the spite RBD of SARS-CoV-2 and that of MERS-CoV (Fig 1C). This shows that SARS coronaviruses taxonomically dissent from MERS coronavirus (Fig 1D).

Given the more than $90 \%$ genomic similarity of SARS-CoV-2 to SARS-CoV, a $25.6 \%$ difference between the structure of the spike RBDs of the SARS-CoVs is relatively significant. This suggests that higher transmission of SARS-CoV-2 than SARS-CoV may be due to a higher binding affinity of SARS-CoV-2 spike RBD to human ACE2. To test this, we aligned the protein structures of SARS-CoV-2 and SARS-CoV spike RBDs interacted with ACE2 by selecting the ACE2 (PDB 6LZG) as reference coordinate to examine whether the RBDs binds to different 
sites of ACE2 or not. Result revealed that both RBDs bind to the same region of ACE2 (Fig 1E). We then, analyzed the binding affinity and interactions between RBD and ACE2 in the CoVs. Result showed that SARS-CoV-2 RBD binds to ACE2 by 12 hydrogen bonds through binding

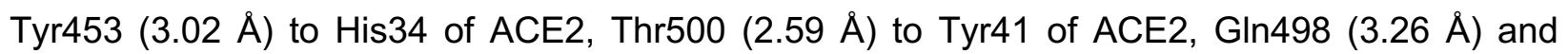

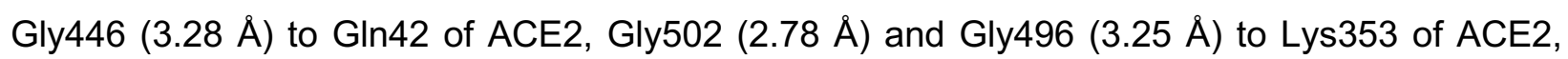
Tyr449 to $\operatorname{Gln} 42(2.84 \AA)$ and Asp38 (2.83 $\AA)$ of ACE2, Lys417 (2.00 $\AA)$ to Asp30 of ACE2, Ala475 (2.81 $\AA$ ) to Ser19 of ACE2 and Asn487 to Gln24 (2.87 $\AA)$ and Tyr83 (2.83 A) of ACE2 (Fig 1F). Nine residues from SARS-CoV-2 RBD including Gln493, Leu455, Asn501, Gly476, Tyr505, Phe456, Tyr480 and Phe486, and 9 residues from ACE2 including Asn330, Arg357, Glu35, Asp355, Leu45, Gly354, Thr27, Phe28 and Met82 were found contributed in the binding by forming hydrophobic contacts (Fig 1F). SARS-CoV RBD binds to ACE2 by 11 hydrogen bonds through binding Asn473 to Thr83 (3.01 $\AA$ ) and Gln24 (2.74 $\AA$ ) of ACE2, Gly488 (2.91 $\AA$ ) and Gly482 (3.35 $\AA$ ) to Lys353 of ACE2, Thr486 with two bonds ( $3.23 \AA$ and $2.42 \AA$ ) to Tyr41 of

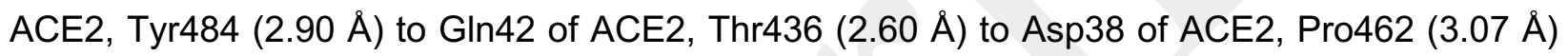
to Ser19 of ACE2 and Arg426 with two bonds (3.11 $\AA$ and $3.26 \AA$ ) to Glu329 of ACE2 (Fig 1G). Seven residues from SARS-CoV RBD including Phe472, Ile489, Tyr491, Tyr487, Leu443, Tyr475 and Phe442, and 11 residues from ACE2 including Met82, Gln235, Gly354, Arg357, Asp355, Asn330, Leu45, Thr27, Phe28, Lys31and Asp30 were found contributed in the binding site by forming hydrophobic contact (Fig 1G).

To investigate the binding affinity of the interactions between RBDs and ACE2, we calculated $\triangle G$ and dissociation constant $\left(K_{d}\right)$ of the interactions. SARS-CoV-2 RBD:ACE2 complex showed a $\Delta G$ of $-12.5 \mathrm{Kcal} / \mathrm{mol}$ and a $K_{d}$ of $7.2 \mathrm{e}-10 \mathrm{~mole}(\mathrm{M})$ (Fig $1 \mathrm{~F}$ ), while, SARS-CoV RBD:ACE2 complex had a $\Delta G$ of $-10.9 \mathrm{Kcal} / \mathrm{mol}$ and a $K_{d}$ of $1.1 \mathrm{e}-8 \mathrm{M}$ (Fig 1G). These results show that the binding affinity of SARS-CoV-2 RBD to ACE2 is higher than that of SARS-CoV RBD to ACE2.

Taken together, there is a significant difference between SARS-CoV-2 and SARS-CoV in terms of spike RBD structure. Despite the structural difference, both the spike RBDs bind to the same region of ACE2. However, SARS-CoV-2 spike RBD binds to ACE2 with higher affinity and form stronger interaction with ACE2 compared to SARS-CoV. 

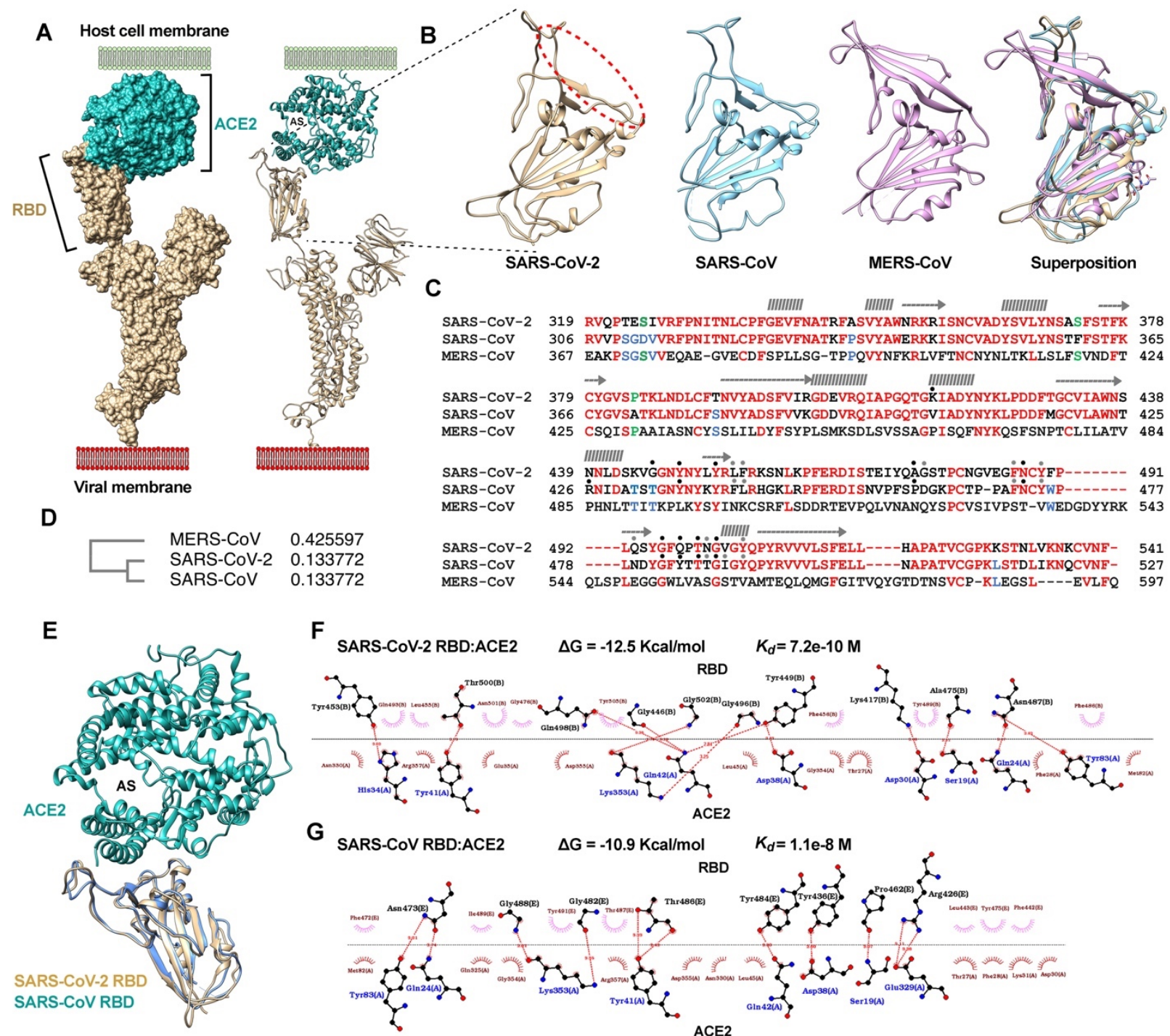

$\mathbf{F}$

SARS-CoV-2 RBD:ACE2 $\quad \Delta G=-12.5 \mathrm{Kcal} / \mathrm{mol} \quad K_{d}=7.2 \mathrm{e}-10 \mathrm{M}$

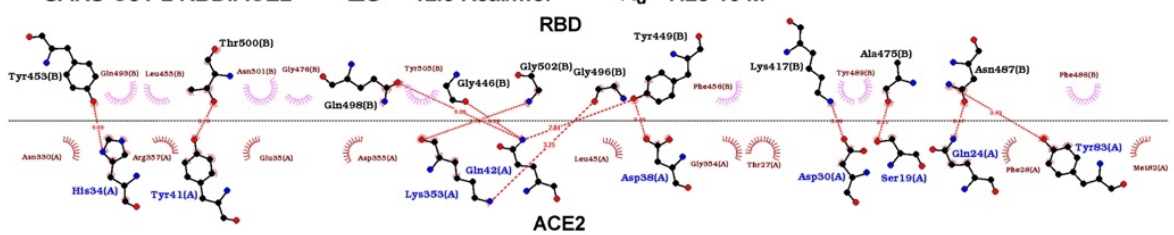

G

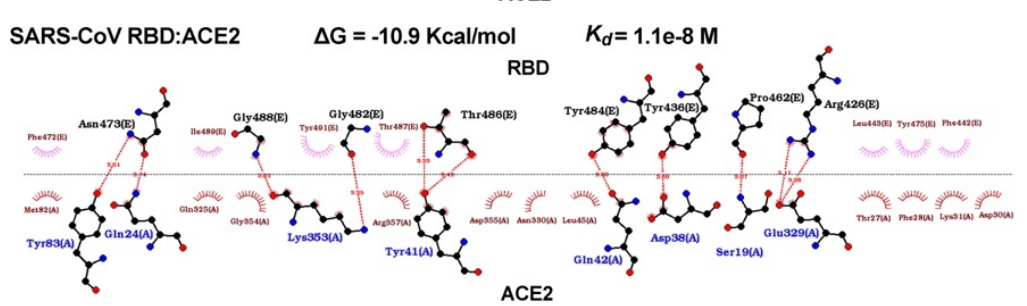

Fig 1. Comparative structure and binding of SARS-CoV-2 and SARS-CoV to Human ACE2. (A) Crystalographic structure of SARS-CoV-2 spike protein (tan) interacted with human ACE2 (seagreen) in the ribbon (right) and surface (left) display (a combination of PDBs 6VSB [15] and 6LZG structures). The open enzymatic active site is illustrated by "AS" (B) Structure of spike RBDs of SARS-CoV-2 (tan; PDB 6LZG), SARS-CoV (aqua; PDB 3SCI), MERS-CoV (pink; 6C6Z [32]) and the superposition display. The binding site to human ACE2 are shown by dashed oval (C) Multiple amino acid sequence alignment of the spike RBDs. The $\alpha$-helices and $\beta$-sheets of SARS-CoV-2 spike RBD are shown by helices and arrows respectively. The common residues between SARS-CoV-2 and SARS-CoV are shown in red letters. The common residues between SARS-CoV-2 and MERS-CoV are shown in green letters. The common residues between SARS-CoV and MERS-CoV are shown in blue letters. Residues 
involved in binding to ACE2 are shown by black (hydrogen bond) and grey (hydrophobic contact) dots on top. (D) Phylogenetic tree and scores of the CoVs. (E) Superposition alignment of crystallographic structures of SARS-CoV-2 (tan) and SARS-CoV (blue) spike RBDs in the interacted form with human ACE2 (seagreen). ( $F$ and $\mathbf{G})$ Residues involved in the interaction of SARS-CoV-2 spike RBD (F) and SARS-CoV spike RBD (G) with human ACE2. The hydrogen bonds and hydrophobic contacts are shown by dashed lines and sunshine respectively.

\section{MLN-4760 has no significant effect on the binding of SARS-CoV-2 spike RBD to ACE2}

\section{S,S)-2-\{1-Carboxy-2-[3-(3,5-dichloro-benzyl)-3H-imidazol-4-YL]-ethylamino\}-4-methyl-} pentanoic acid also named as MLN-4760 with a molecular formula of $\mathrm{C}_{19} \mathrm{H}_{23} \mathrm{C}_{12} \mathrm{~N}_{3} \mathrm{O}_{4}$ (Fig 2C) and a molecular weight of $428.310 \mathrm{Da}$ (PubChem ID: 448281, DrugBank ID: DB12271) is a selective and highly potent inhibitor of human ACE2 carboxypeptidase activity with an IC 50 value of $0.44 \mathrm{nM}[28,33]$. To investigate the effect of MLN-4760 binding to ACE2 on the interaction of SARS-CoV-2 spike RBD with ACE2 we docked the RBD (PDB 6LZG) to human native ACE2 (PDB 1R42) and the ACE2 complexed with MLN-4760 (PDB 1R4L) by using the coordinate of native human ACE2 from 6LZG complex (native ACE2 in complex with SARS-CoV-2 RBD). As shown in Fig 2A, the enzymatic active site of ACE2(native) was open, while binding MLN-4760 to the active site of ACE2 significantly altered the topology of ACE2 structure to a more condensed form, and closed the enzymatic active site (Fig 2B). Protein-ligand interaction analysis of the ACE2(MLN-4760) revealed that MLN-4760 strongly bonded to ACE2 through 10 hydrogen bonds with residues Arg273 (2.74 and $2.93 \AA$ ), His345 (2.66 $\AA$ ), Phe346 (3.09 $\AA)$,

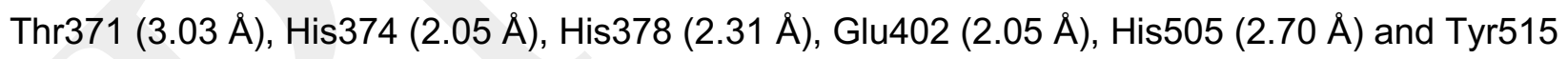
(3.24 A), 4 hydrophobic contacts with residues of Glu375, Phe504, Tyr510 and Arg514, and 2 bonds with $\mathrm{Zn}$ of ACE2 (Figs $2 \mathrm{D}$ and $\mathrm{E}$ ). The $\Delta \mathrm{G}$ of the interaction was calculated as -9.7 $\mathrm{Kcal} / \mathrm{mol}$. 

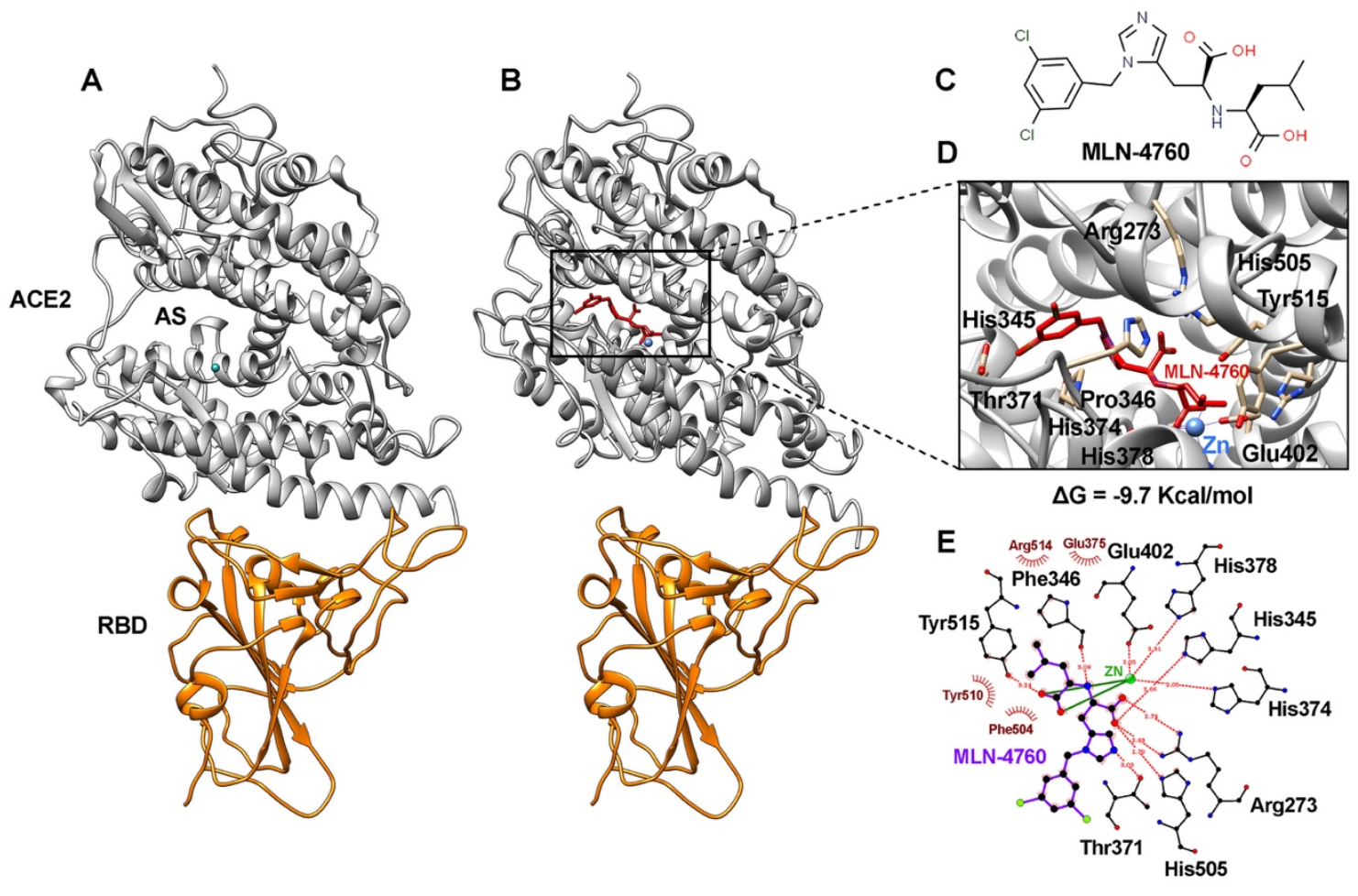

Fig 2. Study model structures. (A and B) Crystalographic structures of SARS-CoV-2 spike protein RBD in complex with ACE2(native) (A) and ACE2(MLN-4760) (B) investigated in this study. The open enzymatic active site is illustrated by "AS". (C) Chemical structure of LMN4760. (D) Interaction of LMN-4760 with the enzymatic active site of human ACE2. (E) Interactions between LMN-4760 and ACE2 at the enzymatic active site of ACE2. The hydrogen bonds and hydrophobic contacts are shown by dashed lines and sunshine respectively.

To study whether MLN-4760 affects the interaction of SARS-CoV-2 spike RBD with ACE2, we investigated the interaction of SARS-CoV-2 spike protein with ACE2 in a native form and in complex with LMN-4760 by a 100 ns MD simulation. The simply root-mean-square deviations (RMSDs) of both RBD:ACE2(native) and RDB:ACE2(MLN-4760) complexes were rapidly stabilized in the first ns of the simulation and were continued in a stable trend during the next 99 ns (Fig 3A). The number of hydrogen bonds at each picosecond (ps) of simulation is shown in Fig 3B. In the first 15 ns the number of hydrogen bonds between RDB and ACE2 in RDB:ACE2(MLN-4760) complex was considerably lower than that of RBD:ACE2(native) complex. This might be due to the different conformation of the ACE2(NLM-4760) structure than that of native ACE2 that in the result of LMN-4760 binding (see Figs $2 A$ and $B$ ). As shown in Fig 
2B, the distance between RBD and ACE2 in RDB:ACE2(MLN-4760) complex was more than that in RDB:ACE2(native) complex. This was because of selecting the spatial coordinate of RBD:ACE2(native) complex as the reference coordinate for docking RDB to ACE2(MLN-4760). After $15 \mathrm{~ns}$, the number of hydrogen bonds of RDB:ACE2(MLN-4760) complex became almost equal to that of RBD:ACE2(native) complex and continued as equal in the next $85 \mathrm{~ns}$ (Fig 3A). These results show that the dynamic and interaction between RBD and ACE2 in RBD:ACE2(MLN-4760) complex is similar to those in RBD:ACE2(native) complex, indicating that RBD binds to ACE2(native) and ACE2(MLN-4760) with a similar affinity.

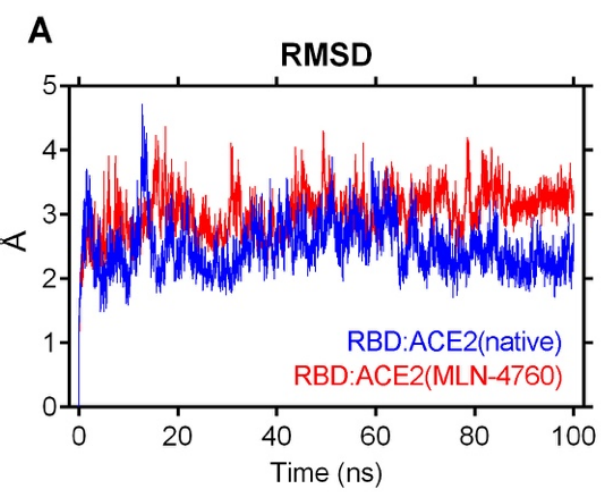

A

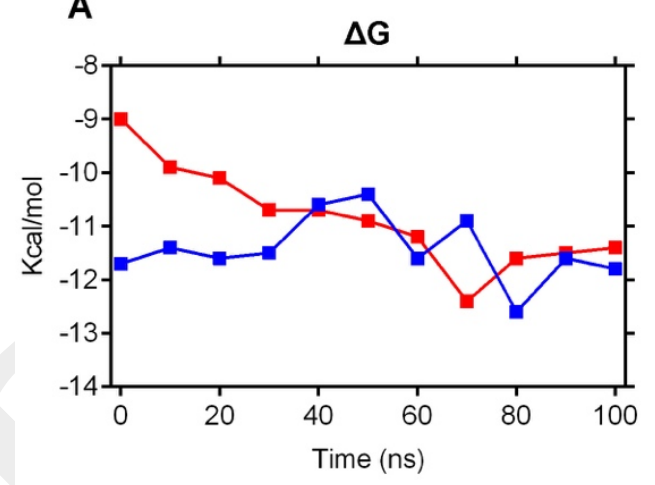

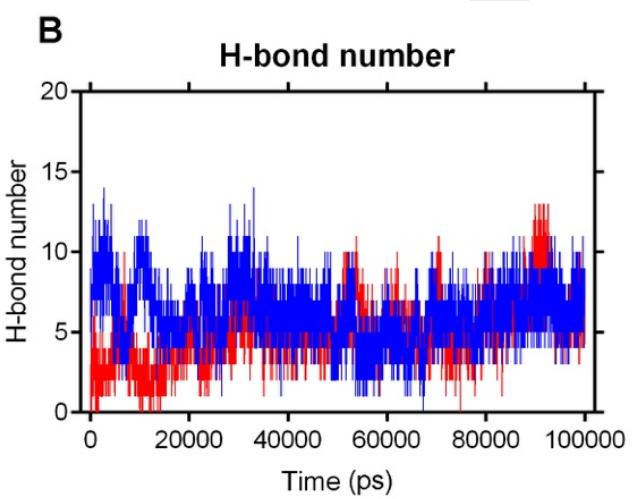

B

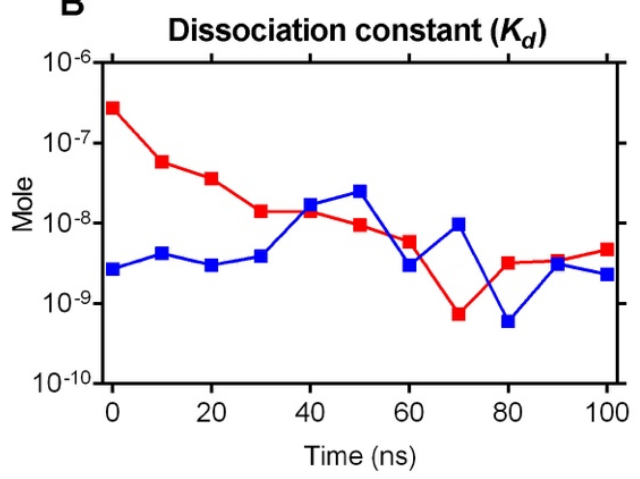

Fig 3. The molecular dynamic simulation results of interaction between SARS-CoV-2 spike RBD and human ACE2 in the native form and inhibited form by MLN-4760. (A) The root-meansquare deviation (RMSD) of SARS-CoV-2 spike RBD in complex with ACE2(native) and ACE2(MLN-4760) at every $1 \mathrm{~ns}$ of the molecular dynamics simulation. (B) the number of hydrogen bonds between the RBD:ACE2 complexes at every 1 ps of the molecular dynamic simulation $(100,000 \mathrm{ps})$. (C) Binding energy $(\Delta G)$ of interaction between the RBD:ACE2 complexes at every $20 \mathrm{~ns}$ of the molecular dynamics simulation. (D) Dissociation constant $\left(K_{d}\right)$ of interaction between the RBD:ACE2 complexes at every 20 ns of the molecular dynamics simulation. 
For further confirmation, we performed a time-lapse analysis interactions between RBD and ACE2 in the complexes at every $10 \mathrm{~ns}$ of simulation. The $\Delta \mathrm{G}$ and $K_{d}$ values of the RBD:ACE2 complex at every $20 \mathrm{~ns}$ are shown in Figs $3 \mathrm{~A}$ and $\mathrm{B}$. At $0 \mathrm{~ns} \Delta \mathrm{G}$ and $K_{d}$ of RBD:ACE2(native) complex was calculated as respectively $-11.7 \mathrm{Kcal} / \mathrm{mol}$ and 2.7e-9 M, while RBD:ACE2(MLN4760) complex revealed a $\Delta \mathrm{G}$ and $K_{d}$ of $-9.9 \mathrm{Kcal} / \mathrm{mol}$ and $2.7 \mathrm{e}-7 \mathrm{M}$ respectively. The values of RBD:ACE2(native) were stable by 30 ns, however, the values of RBD:ACE2(MLN-4760) complex were reduced to be almost equal to those of RBD:ACE2(native) complex at $40 \mathrm{~ns}$. This is due to the more distance of ACE2(MLN-4760) structure from RBD at the start time of simulation as discussed above. The highest binding affinity (indicated by the lowest $\Delta G$ and $K_{d}$ values) of RBD:ACE2(native) complex was observed at 80 ns of simulation ( $\triangle \mathrm{G}=-12.6$ $\left.\mathrm{Kcal} / \mathrm{mol} ; K_{d}=6.9 \mathrm{e}-10 \mathrm{M}\right)$. While the highest binding affinity of RBD:ACE2(MLN-4760) complex was detected at approximately $70 \mathrm{~ns}$ of the simulation $\left(\Delta \mathrm{G}=-10.9 \mathrm{Kcal} / \mathrm{mol} ; K_{d}=9.7 \mathrm{e}-9 \mathrm{M}\right)$ (Figs $3 A$ and $B$ ). The lowest binding affinity of RBD:ACE2(native) complex was detected at 50 ns $\left(\Delta G=-10.4 \mathrm{Kcal} / \mathrm{mol} ; K_{d}=2.5 \mathrm{e}-8 \mathrm{M}\right)$, and lowest binding affinity of RBD:ACE2(MLN-4760) was deleted at $0 \mathrm{~ns}$ of simulation as mentioned above (Figs $3 \mathrm{~A}$ and $\mathrm{B}$ ). At the end of $100 \mathrm{~ns}$ simulation the binding affinities of RBD with native ACE2 and ACE2(MLN-4760) were similar to each other ( $\Delta \mathrm{G}$ of -11.8 versus $-11.4 \mathrm{Kcal} / \mathrm{mol}$ and $K_{d}$ of $2.3 \mathrm{e}-9$ versus $4.7 \mathrm{e}-09 \mathrm{M}$ ).

We also investigated the time-laps binding sites in terms of hydrogen bonds and hydrophobic contacts at every $20 \mathrm{~ns}$ of the simulation as shown in Fig 4. At $100 \mathrm{~ns}$ simulation, the RBD was found bonded to native ACE2 by 7 hydrogen bonds through binding Ala475 (2.76 $\AA$ ) to Ser19 of ACE2, Glu484 (2.79 A) to Lys31 of ACE2, Thr500 (2.93 Å) to Asp355 of ACE2, Gln493 (2.90 A)

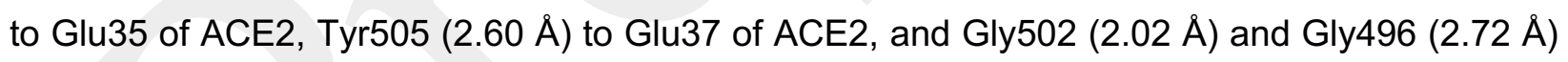
to Lys353 of ACE2. Residues Gly475, Tyr489, Phe456, Tyr473, Phe486, Leu455, Tyr453, Asn501 and Gln498 from RBD and Gln24, Phe28, Thr27, Tyr83, Leu79, Asn330, His34, Gly354 and Tur41 from ACE2 were involved in the interaction by forming hydrophobic contacts (Fig 4). At 100 ns simulation, the RBD was detected bonded to ACE2(MLN-4760) by 9 hydrogen bonds

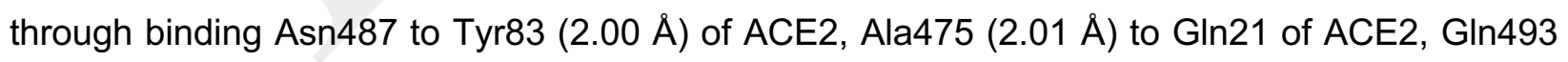
to Glu35 (2.5 $\AA$ ) and Lys31 (2.77 $\AA$ ) of ACE2, Gly496 (2.99 $\AA$ ) and Gly502 (2.23 $\AA$ ) to Lys353 of ACE2, Gln498 (3.31 $\AA$ ) to Gln42 of ACE2 and Thr500 (2.00 $)$ to Tyr41 and Asp355 (2.09 $\AA)$ of ACE2. Residues Phe486, Leu455, Tyr489, Phe456, Tyr505, Asn501, Tyr449, Arg403, Tyr453 and Lys417 from RBD and Leu79, Met82, Asp30, Phe28, Thr27, Asp38, Gly354, Asn330 and His34 from ACE2(MLN-4760) were found involved in the interaction by forming hydrophobic contacts (Fig 4). 


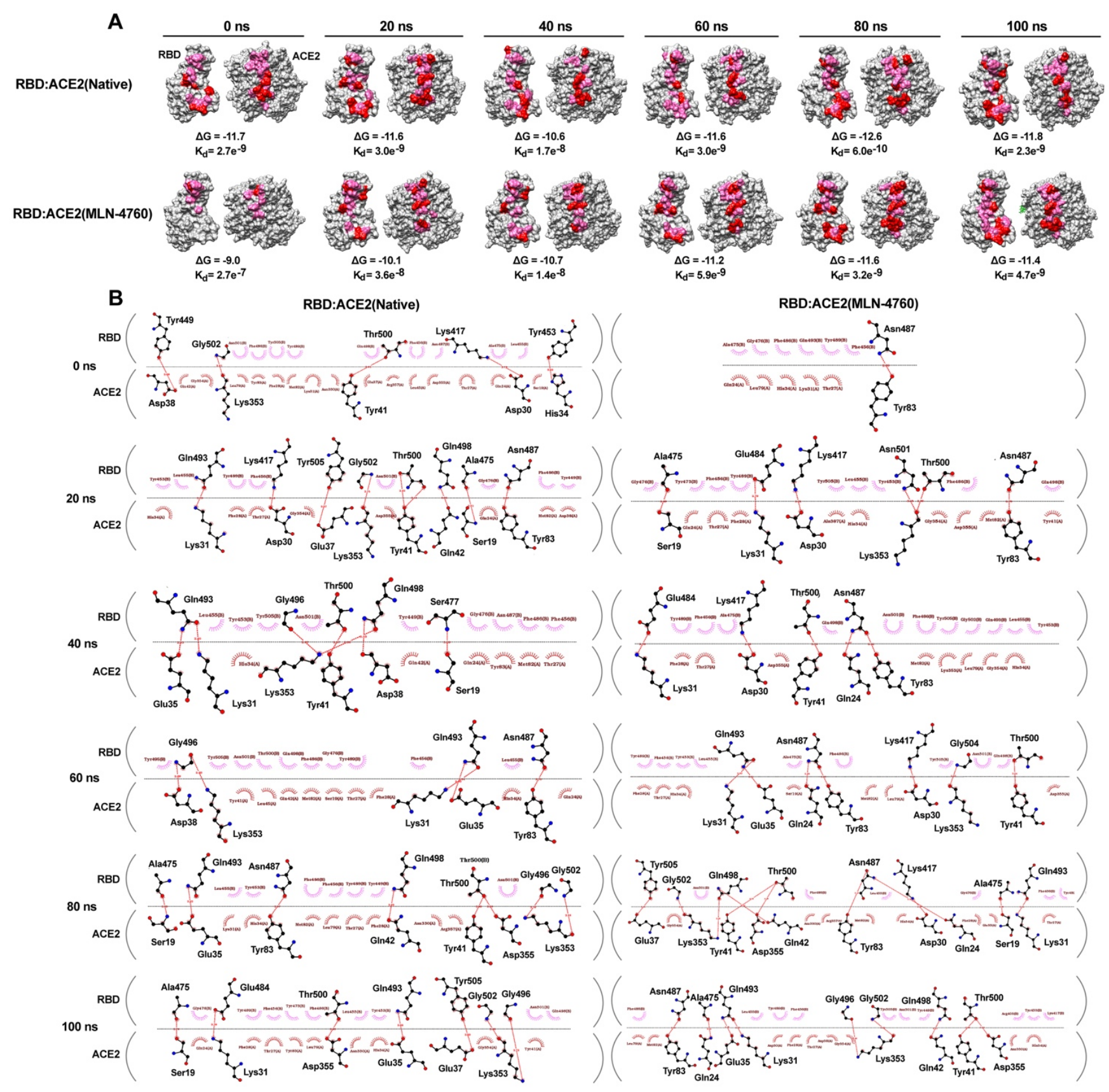

Fig 4. Time-laps dynamics and interaction of SARS-CoV-2 spike RBD with human ACE2 in the native form and inhibited form by MLN-4760. (A) Binding sites of SARS-CoV-2 spike RBD (left) and human ACE2 (right) and their binding values in RBD:ACE2(native) and RBD:ACE2(MLN-4760) complexes at every 20 ns of the molecular dynamics simulation. (B) Residues involved in the interaction of SARS-CoV-2 spike RBD with human ACE2 in RBD:ACE2(native) (left column) and RBD:ACE2(MLN-4760) (right column) complexes at 
every 20 ns of the molecular dynamics simulation. The hydrogen bonds and hydrophobic contacts are shown by dashed lines and sunshine respectively.

Taken together, these results show that RBD binding to ACE2(native) and ACE2(MLN-4760) takes place with a similar dynamic and binding affinity. These results indicate that inhibition of ACE2 by MLN-4760 has no significant effect on the binding of SARS-CoV-2 spike RBD to the ACE2 receptor.

\section{Binding SARS-CoV-2 spike RBD to ACE2(MLN-4760) abrogates the inhibitory effect of MLN-4760}

Structural analysis revealed no major change in the conformation of ACE2(native) in the result of the 100 ns simulation (Fig 5A). However, ACE2(MLN-4760) underwent a major conformation change in the result of $100 \mathrm{~ns}$ simulation compared to the structures at 0 ns (Fig $5 B)$. The conformation of ACE2(MLN-4760) at 100 ns simulation was found highly similar to that of ACE2(native) with open enzymatic active site (Figs 5B and E). Interestingly, in the result of 100 ns simulation, MLN-4760 was found removed from the enzymatic active site of the ACE2 and bonded to an outer region of the ACE2 (Asn121, Ile126 and GIn175) by hydrophobic contacts with a relatively lower affinity $(\Delta G=-6.4 \mathrm{Kcal} / \mathrm{mol}$ versus $-9.7 \mathrm{Kcal} / \mathrm{mol})$ (Figs $5 B-D)$. Time-laps simulation analysis revealed that the MLN-4760 removal had occurred in the first 10 ns of simulation. This phenomenon might be due to the binding of RBD to the ACE2(MLN4760).

Taken together, these results show that binding SARS-CoV-2 spike RBD to the ACE2 complexed with MLN-4760 abrogates the inhibitory effect of MLN-4760 and rescues the enzymatic activity of ACE2 by removing MLN-4760 from ACE2 enzymatic active site. 

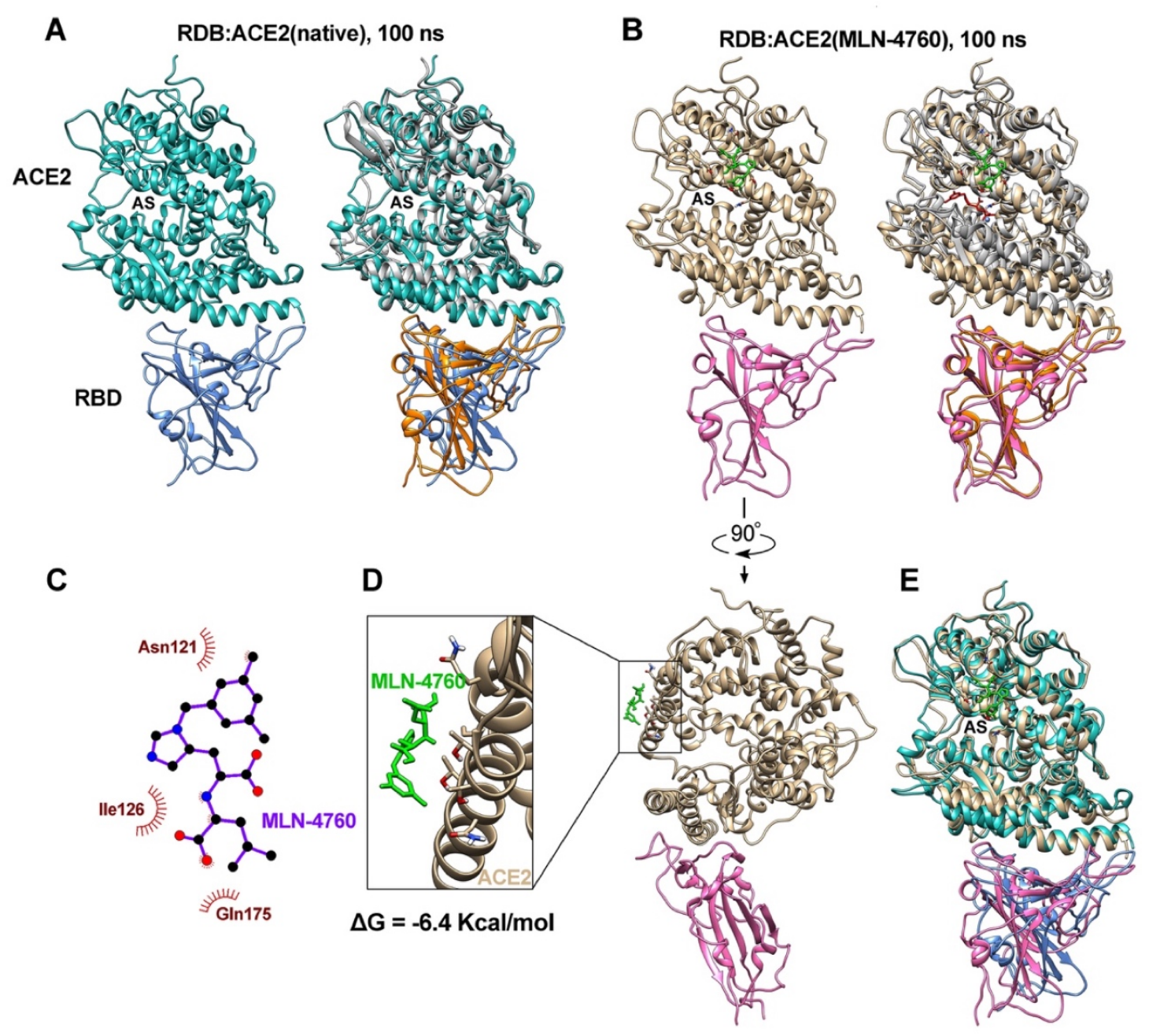

Fig 5. Structure of SARS-CoV-2 spike protein in complex with human ACE2(native) and ACE2(MLN-5760) after $100 \mathrm{~ns}$ molecular dynamics simulation. The open enzymatic active site is illustrated by "AS". (A and B) Structure of RBD:ACE2(native) (A) and RBD:ACE2(MLN4760) (B) complexes after $100 \mathrm{~ns}$ molecular dynamics simulation (left) and in superposition with the initial ( $0 \mathrm{~ns}$ ) structure (right). (C and D) Position and the residues involved in the secondary binding site of MLN-5760 in the RBD:ACE2(MLN-4760) complexes after $100 \mathrm{~ns}$ molecular dynamic simulation. The hydrophobic contacts are shown by sunshine. $(E)$ Superposition display of the RBD:ACE2(native) (RBD in blue and ACE2 in seagreen) and RBD:ACE2(MLN-4760) (RBD in pink and ACE2 in tan) after 100 ns molecular dynamics simulation. 


\section{Acknowledgments}

This is a preprint manuscript for peer-review. All materials are sunjected to copyright license Attribution-NonCommercial-NoDerivatives 4.0 International (CC BY-NC-ND 4.0). This study was not financially supported by any institution or agency. The authors are thankful to Drs. Robin Clugston and Oana Caluseru of the University of Alberta for supporting this study. The authors also thank Compute Canada Support Team for technical support and follow up.

\section{Author Contributions}

All authors have read and approved the manuscript. B.N. designed and led the project, and participated in the generating structural models, MD simulation, data analysis, and writing manuscript. A.G. participated in MD simulation, data analysis, and writing manuscripts. K.G. and N.N. participated in the literature review, data analysis, and writing manuscript.

\section{Conflicts of Interest}

The authors declare no conflict of interest.

\section{References}

1. Wu Z, McGoogan JM. Characteristics of and Important Lessons From the Coronavirus Disease 2019 (COVID-19) Outbreak in China: Summary of a Report of 72314 Cases From the Chinese Center for Disease Control and Prevention. JAMA. 2020. doi:10.1001/jama.2020.2648

2. Hui DS, I Azhar E, Madani TA, Ntoumi F, Kock R, Dar O, et al. The continuing 2019nCoV epidemic threat of novel coronaviruses to global health - The latest 2019 novel coronavirus outbreak in Wuhan, China. International journal of infectious diseases : IJID : official publication of the International Society for Infectious Diseases. Canada; 2020. pp. 264-266. doi:10.1016/j.ijid.2020.01.009

3. Zhu N, Zhang D, Wang W, Li X, Yang B, Song J, et al. A Novel Coronavirus from Patients with Pneumonia in China, 2019. N Engl J Med. 2020;382: 727-733. doi:10.1056/NEJMoa2001017

4. The species Severe acute respiratory syndrome-related coronavirus: classifying 2019nCoV and naming it SARS-CoV-2. Nat Microbiol. 2020;5: 536-544. doi:10.1038/s41564- 
020-0695-z

5. Huang C, Wang Y, Li X, Ren L, Zhao J, Hu Y, et al. Clinical features of patients infected with 2019 novel coronavirus in Wuhan, China. Lancet (London, England). 2020;395: 497-506. doi:10.1016/S0140-6736(20)30183-5

6. Lescure F-X, Bouadma L, Nguyen D, Parisey M, Wicky P-H, Behillil S, et al. Clinical and virological data of the first cases of COVID-19 in Europe: a case series. Lancet Infect Dis. 2020. doi:10.1016/S1473-3099(20)30200-0

7. Yu N, Li W, Kang Q, Xiong Z, Wang S, Lin X, et al. Clinical features and obstetric and neonatal outcomes of pregnant patients with COVID-19 in Wuhan, China: a retrospective, single-centre, descriptive study. Lancet Infect Dis. 2020. doi:10.1016/S14733099(20)30176-6

8. Wang Z, Yang B, Li Q, Wen L, Zhang R. Clinical Features of 69 Cases with Coronavirus Disease 2019 in Wuhan, China. Clin Infect Dis. 2020. doi:10.1093/cid/ciaa272

9. WHO. WHO Director-General's opening remarks at the media briefing on COVID-19-11 March 2020. In: The World Health Organization [Internet]. 2020 [cited 20 Mar 2020]. Available: https://www.who.int

10. WHO. Coronavirus disease (COVID-2019) situation reports. 2020 [cited 20 Apr 2020]. Available: https://www.who.int/emergencies/diseases/novel-coronavirus-2019/situationreports

11. Andersen KG, Rambaut A, Lipkin WI, Holmes EC, Garry RF. The proximal origin of SARS-CoV-2. Nature medicine. United States; 2020. pp. 450-452. doi:10.1038/s41591020-0820-9

12. Forster P, Forster L, Renfrew C, Forster M. Phylogenetic network analysis of SARS-CoV2 genomes. Proc Natl Acad Sci U S A. 2020. doi:10.1073/pnas.2004999117

13. Wu C, Liu Y, Yang Y, Zhang P, Zhong W, Wang Y, et al. Analysis of therapeutic targets for SARS-CoV-2 and discovery of potential drugs by computational methods. Acta Pharmaceutica Sinica. B. 2020. doi:10.1016/j.apsb.2020.02.008

14. Walls AC, Park Y-J, Tortorici MA, Wall A, McGuire AT, Veesler D. Structure, Function, and Antigenicity of the SARS-CoV-2 Spike Glycoprotein. Cell. 2020. doi:10.1016/j.cell.2020.02.058

15. Wrapp D, Wang N, Corbett KS, Goldsmith JA, Hsieh C-L, Abiona O, et al. Cryo-EM 
structure of the 2019-nCoV spike in the prefusion conformation. Science. 2020;367: 1260-1263. doi:10.1126/science.abb2507

16. Chaudhury A, Hussey GS, Ray PS, Jin G, Fox PL, Howe PH. TGF-beta-mediated phosphorylation of hnRNP E1 induces EMT via transcript-selective translational induction of Dab2 and ILEI. Nat Cell Biol. 2010;12: 286-293. doi:10.1038/ncb2029

17. Lam TT-Y, Shum MH-H, Zhu H-C, Tong Y-G, Ni X-B, Liao Y-S, et al. Identifying SARSCoV-2 related coronaviruses in Malayan pangolins. Nature. 2020. doi:10.1038/s41586020-2169-0

18. Cyranoski D. Mystery deepens over animal source of coronavirus. Nature. England; 2020. pp. 18-19. doi:10.1038/d41586-020-00548-w

19. Zhang $T, W u Q$, Zhang Z. Probable Pangolin Origin of SARS-CoV-2 Associated with the COVID-19 Outbreak. Curr Biol. 2020;30: 1346-1351.e2. doi:10.1016/j.cub.2020.03.022

20. Benvenuto D, Giovanetti M, Ciccozzi A, Spoto S, Angeletti S, Ciccozzi M. The 2019-new coronavirus epidemic: Evidence for virus evolution. J Med Virol. 2020;92: 455-459. doi:10.1002/jmv.25688

21. Zhou P, Yang X-L, Wang X-G, Hu B, Zhang L, Zhang W, et al. A pneumonia outbreak associated with a new coronavirus of probable bat origin. Nature. 2020;579: 270-273. doi:10.1038/s41586-020-2012-7

22. Hoffmann M, Kleine-Weber H, Schroeder S, Kruger N, Herrler T, Erichsen S, et al. SARS-CoV-2 Cell Entry Depends on ACE2 and TMPRSS2 and Is Blocked by a Clinically Proven Protease Inhibitor. Cell. 2020. doi:10.1016/j.cell.2020.02.052

23. Li W, Moore MJ, Vasilieva N, Sui J, Wong SK, Berne MA, et al. Angiotensin-converting enzyme 2 is a functional receptor for the SARS coronavirus. Nature. 2003;426: 450-454. doi:10.1038/nature02145

24. Tipnis SR, Hooper NM, Hyde R, Karran E, Christie G, Turner AJ. A human homolog of angiotensin-converting enzyme. Cloning and functional expression as a captoprilinsensitive carboxypeptidase. J Biol Chem. 2000;275: 33238-33243. doi:10.1074/jbc.M002615200

25. Paz Ocaranza M, Riquelme JA, Garcia L, Jalil JE, Chiong M, Santos RAS, et al. Counterregulatory renin-angiotensin system in cardiovascular disease. Nat Rev Cardiol. 2020;17: 116-129. doi:10.1038/s41569-019-0244-8 
26. Kreutz R, Algharably EAE-H, Azizi M, Dobrowolski P, Guzik T, Januszewicz A, et al. Hypertension, the renin-angiotensin system, and the risk of lower respiratory tract infections and lung injury: implications for COVID-19. Cardiovasc Res. 2020. doi:10.1093/cvr/cvaa097

27. Huentelman MJ, Zubcevic J, Hernandez Prada JA, Xiao X, Dimitrov DS, Raizada MK, et al. Structure-based discovery of a novel angiotensin-converting enzyme 2 inhibitor. Hypertens (Dallas, Tex 1979). 2004;44: 903-906. doi:10.1161/01.HYP.0000146120.29648.36

28. Towler P, Staker B, Prasad SG, Menon S, Tang J, Parsons T, et al. ACE2 X-ray structures reveal a large hinge-bending motion important for inhibitor binding and catalysis. J Biol Chem. 2004;279: 17996-18007. doi:10.1074/jbc.M311191200

29. Van Der Spoel D, Lindahl E, Hess B, Groenhof G, Mark AE, Berendsen HJC. GROMACS: fast, flexible, and free. J Comput Chem. 2005;26: 1701-1718. doi:10.1002/jcc.20291

30. Best RB, Zhu X, Shim J, Lopes PEM, Mittal J, Feig M, et al. Optimization of the additive CHARMM all-atom protein force field targeting improved sampling of the backbone $\varphi, \Psi$ and side-chain $X(1)$ and $X(2)$ dihedral angles. J Chem Theory Comput. 2012;8: 32573273. doi:10.1021/ct300400x

31. Gautieri A, Vesentini S, Montevecchi FM, Redaelli A. Mechanical properties of physiological and pathological models of collagen peptides investigated via steered molecular dynamics simulations. J Biomech. 2008;41: 3073-3077. doi:10.1016/j.jbiomech.2008.06.028

32. Wang L, Shi W, Chappell JD, Joyce MG, Zhang Y, Kanekiyo M, et al. Importance of Neutralizing Monoclonal Antibodies Targeting Multiple Antigenic Sites on the Middle East Respiratory Syndrome Coronavirus Spike Glycoprotein To Avoid Neutralization Escape. J Virol. 2018;92. doi:10.1128/JVI.02002-17

33. Dales NA, Gould AE, Brown JA, Calderwood EF, Guan B, Minor CA, et al. Substratebased design of the first class of angiotensin-converting enzyme-related carboxypeptidase (ACE2) inhibitors. J Am Chem Soc. 2002;124: 11852-11853. doi:10.1021/ja0277226 